\title{
S6K1 inhibition enhances the apoptotic cell death of breast cancer cells in response to $\mathrm{Bcl}-2 / \mathrm{Bcl}-\mathrm{xL}$ inhibition by the downregulation of survivin
}

\author{
JIN-AH PARK ${ }^{1,5}$, HYEON-OK JIN ${ }^{1}$, HA-NA LEE ${ }^{1}$, JIN-HEE KIM $^{1}$, IN-CHUL PARK ${ }^{2}$, WOO CHUL NOH ${ }^{3}$, \\ YOON HWAN CHANG ${ }^{4}$, YOUNG JUN HONG ${ }^{4}$, KEUN-CHUL KIM $^{5}$ and JIN KYUNG LEE ${ }^{1,4}$
}

\author{
${ }^{1}$ KIRAMS Radiation Biobank, Korea Institute of Radiological and Medical Sciences; ${ }^{2}$ Division of Radiation Cancer Research, \\ Korea Institute of Radiological and Medical Sciences; Departments of ${ }^{3}$ Surgery and ${ }^{4}$ Laboratory Medicine, \\ Korea Cancer Center Hospital, Korea Institute of Radiological and Medical Sciences, Seoul 139-706; \\ ${ }^{5}$ Department of Biological Sciences, College of Natural Sciences, \\ Kangwon National University, Chuncheon 200-701, Republic of Korea
}

Received September 3,2014; Accepted April 27, 2015

DOI: $10.3892 / 01.2015 .3369$

\begin{abstract}
Breast cancer cells possess a deregulated apoptotic pathway with increased expression levels of anti-apoptotic B-cell lymphoma-2 (Bcl-2) family proteins and ribosomal S6 kinase 1 (S6K1) protein activity. Therefore, combined interference of anti-apoptotic Bcl-2 family and S6K1 protein expression may be a reasonable therapeutic strategy for the treatment of patients with breast cancer. In the present study, it was identified that the administration of a combination of ABT263 [navitoclax; a Bcl-2/Bcl-extra large (Bcl-xL) inhibitor] and PF4708671 (an S6K1 inhibitor) markedly increased apoptotic cell death in the BT474 breast cancer cells compared with the administration of either agent alone. Furthermore, the downregulation of $\mathrm{Bcl}-2 / \mathrm{Bcl}-\mathrm{xL}$ and S6K1 with small interfering RNA induced a significant increase in cell death compared with RNA interference of either agent alone. Notably, combination treatment with ABT263 and PF4708671 decreased the expression level of survivin protein, with this ectopic expression of survivin attenuating cell death. Thus, the present study determined that the combined inhibition of Bcl-2/Bcl-xL and S6K1 may be a good strategy for treating patients with breast cancer.
\end{abstract}

\section{Introduction}

All types of cancer cell are prone to resisting apoptotic cell death; therefore, knowledge of the mechanisms of resistance to apoptosis may lead to the development of novel therapeutic strategies (1). Changes in the apoptotic susceptibility

Correspondence to: Dr Jin Kyung Lee, KIRAMS Radiation Biobank, Korea Institute of Radiological and Medical Sciences, 75 Nowon-ro, Nowon, Seoul 139-706, Republic of Korea

E-mail: jklee@kirams.re.kr

Key words: B-cell lymphoma-extra large, B-cell lymphoma-2, breast cancer, survivin, ribosomal S6 kinase 1 of cancer cells may contribute to enhanced resistance to conventional anticancer therapies, such as radiation and cytotoxic agents, as well as to neoplastic development (2). Altered expression of B-cell lymphoma-2 (Bcl-2) family members has been proposed as a possible mechanism for the development of resistance to certain cytotoxic antineoplastic agents. The Bcl-2 family of proteins is composed of pro- and anti-apoptotic members that regulate cellular proliferation and death, respectively, via inter- and intra-family interactions $(3,4)$. In particular, Bcl-2 has emerged as an important clinical prognostic marker in breast cancer $(5,6)$, being overexpressed in $\sim 75 \%$ of cases of breast cancer (approaching $85 \%$ for estrogen-positive breast cancer) (6). ABT263 (navitoclax) is a small molecule that binds with high affinity to Bcl-2 and Bcl-extra large (Bcl-xL), and antagonizes their anti-apoptotic functions, thereby inducing apoptosis in various cancer cell types $(7,8)$. However, this molecule is limited by its lack of efficacy, and its significant toxicity and resistance (9).

Ribosomal S6 kinase 1 (S6K1) is the downstream effector of mammalian target of rapamycin complex 1 (mTORC1), a protein complex that regulates essential cellular functions, including cell survival, proliferation, metabolism, migration and angiogenesis (10). The S6K1 gene is amplified in numerous breast cancer cell lines (11-13), and S6K1 gene amplification or protein expression has been associated with a poor prognosis in breast cancer (14). Thus, the targeting of S6K1 in combination with ABT263 may present an effective strategy for the treatment of patients with breast cancer.

Therefore, the present study aimed to examine the combinatorial apoptotic effects of ABT263 (a Bcl-2/Bcl-xL inhibitor) and PF4708671 (an S6K1 inhibitor) treatment on BT474 breast cancer cells.

\section{Materials and methods}

Cell culture and reagents. The breast cancer BT474 and MCF7 cell lines were obtained from the American Type 
Culture Collection (Manassas, VA, USA) and cultured in RPMI-1640 medium and Dulbecco's modified Eagle's medium (Invitrogen Life Technologies, Carlsbad, CA, USA) containing 10\% fetal bovine serum (Invitrogen Life Technologies). PF4708671 was purchased from Sigma-Aldrich (St. Louis, MO, USA), ABT263 (navitoclax) from Selleckchem (Houston, TX, USA) and Sorafenib from Bayer Healthcare Pharmaceuticals (New Haven, CT, USA).

Assessment of cell death. The BT474 cells were treated with $0,0.5,1$ and $2 \mu \mathrm{M} \mathrm{ABT} 263$, or $0,5,10$ and $20 \mu \mathrm{M}$ PF4708671. The treated cells were stained with Annexin V-fluorescein isothiocyanate (FITC) and propidium iodide (PI; BD Biosciences, San Jose, CA, USA) for the assessment of apoptotic cell death, as previously described (15). As a positive control, MCF7 cells were treated with $5 \mu \mathrm{M}$ sorafenib and deprived of glucose for $24 \mathrm{~h}$.

Western blot analysis. The cells were lysed in lysis buffer [50 mM Tris- $\mathrm{HCl}$ (pH 7.5), $150 \mathrm{mM} \mathrm{NaCl}, 1 \% \mathrm{NP}-40,0.5 \%$ sodium deoxycholate and $0.1 \%$ SDS] supplemented with a protease inhibitor cocktail (Roche Diagnostics $\mathrm{GmbH}$, Mannheim, Germany). The protein concentrations were then measured using the Bradford method (16). The cell lysates were separated via SDS-PAGE and transferred to nitrocellulose membranes, followed by immunoblotting with specific primary and horseradish peroxidase-conjugated secondary antibodies. Antibodies specific for cleaved poly(ADP-ribose) polymerase (PARP; rabbit polyclonal; 1:1,000; cat no. 9541), p-S6 at Ser240/244 (rabbit monoclonal; 1:2,000; cat no. 4838), S6K1 (rabbit polyclonal; cat no. 9202; 1:1,000) and survivin(rabbitmonoclonal; 1:1,000; catno.2808)wereobtained from Cell Signaling Technology (Beverly, MA, USA), an antibody for Bcl-xL (mouse monoclonal; 1:1,000; cat no. 610746) was obtained from BD Biosciences (Franklin Lakes, NJ,USA), and an antibody for $\beta$-actin (mouse monoclonal; 1:5,000; cat no. A5316) was obtained from Sigma-Aldrich. The immunoreactive bands were visualized using SuperSignal West Pico Chemiluminescent Substrates (Thermo Scientific Pierce, Rockford, IL, USA).

small interfering (siRNA) and transfection. Bcl-2 (cat no. sc-29214), Bcl-xL (cat no. sc-43630) and control (cat no. sc-37007) siRNAs were purchased from Santa Cruz Biotechnology, Inc. (San Jose, CA, USA). S6K1 (cat no. SI00301721) and control siRNAs (cat no. 1022076) were purchased from Qiagen, Inc. (Valencia, CA, USA). Transient 24-h siRNA transfections were performed with Lipofectamine ${ }^{\circledR}$ RNAiMAX reagent, according to the manufacturer's instructions (Invitrogen Life Technologies). The BT474 cells were transiently transfected with the indicated siRNAs for $24 \mathrm{~h}$.

Measurement of cell viability. The cell viability was determined by measuring the mitochondrial conversion of 3-(4,5-dimethylthiazolyl-2)-2,5-diphenyltetrazolium bromide to a colored product, as previously described (15). Images of cell populations were captured microscopically with an Olympus CKX41 microscope (Olympus Corporation, Tokyo, Japan).
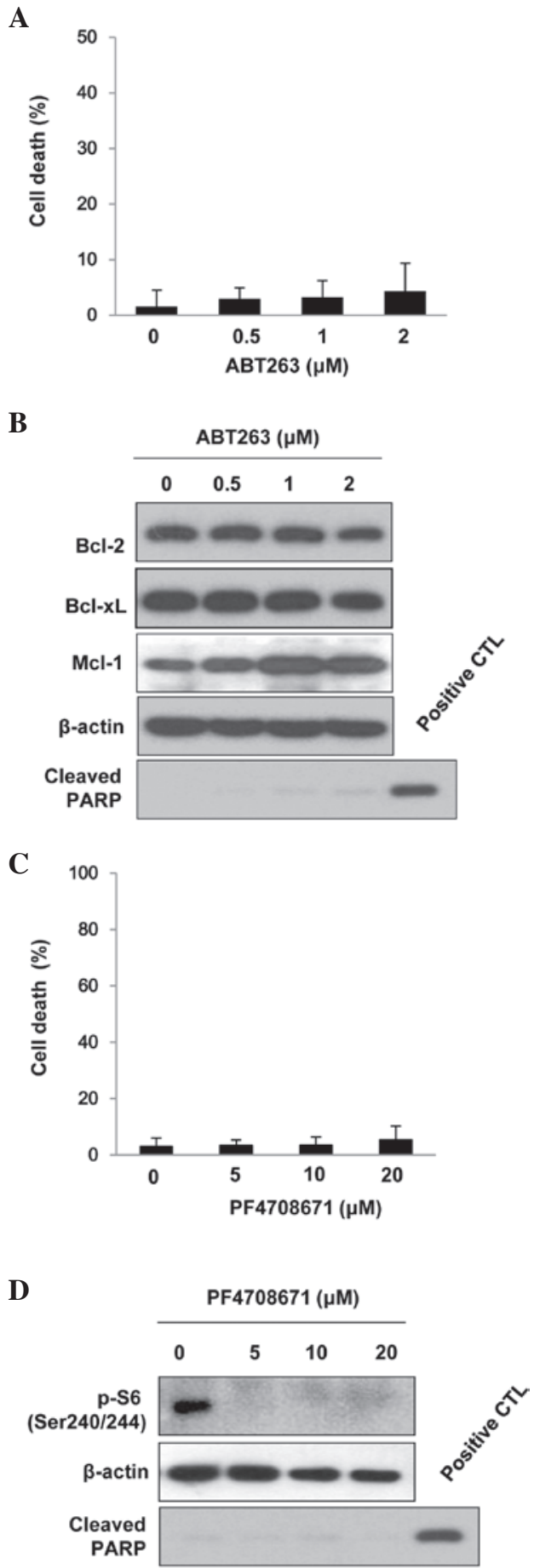

Figure 1. (A and B) ABT263 and (C and D) PF4708671 had no effect on BT474 cell death when applied individually. BT474 cells were treated with the indicated concentrations of ABT263 or PF4708671 for $24 \mathrm{~h}$. (A and D) Cell death was determined using flow cytometry following Annexin V/propidium iodide staining. Data are presented as the means of triplicate samples with error bars indicating the standard deviation from the mean. (B and C) Protein expression levels were estimated using western blot analysis. As a positive CTL, MCF7 cells were treated with $5 \mu \mathrm{M}$ sorafenib and deprived of glucose for $24 \mathrm{~h}$. Bcl-2, B-cell lymphoma-2; Bcl-xl, B-cell lymphoma-extra large; PARP, poly(ADP-ribose) polymerase; CTL, control.

$R N A$ isolation and reverse transcription-polymerase chain reaction $(R T-P C R)$ analysis. RNA isolation and RT-PCR analysis were conducted as previously described (17). The following primers were used: 
A

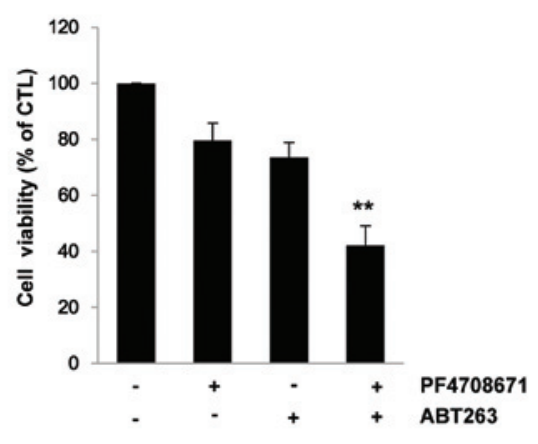

C

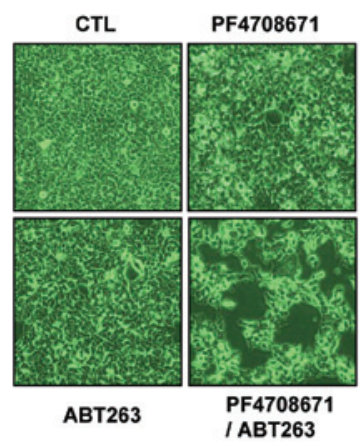

B

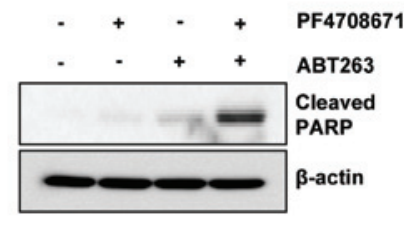

D

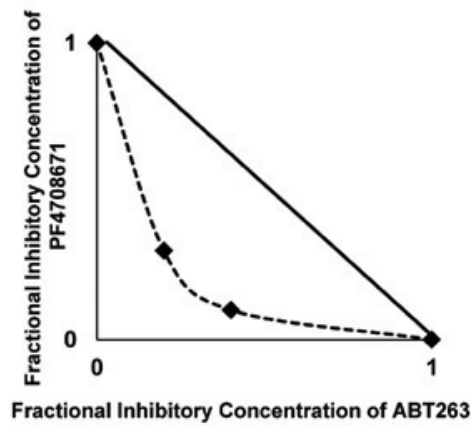

Figure 2. Combined treatment with PF4708671 and ABT263 synergistically induces cell death. (A-C) BT474 cells were treated with or without $1 \mu$ M ABT263 and/or $10 \mu \mathrm{M}$ PF4708671 for $24 \mathrm{~h}$. (A) Cell viability was determined by performing a 3-(4,5-dimethylthiazolyl-2)-2,5-diphenyltetrazolium bromide assay. Data are presented as the means of triplicate samples, with error bars indicating the standard deviation from the mean. (B) Protein expression levels of cleaved PARP were analyzed by western blotting. (C) Images of cell populations for each treatment group were obtained by fluorescence microscopy. (D) Synergy between the effects of PF4708671 and ABT263 was assessed by an isobologram analysis. ${ }^{* *} \mathrm{P}<0.01$ vs. PF4708671- or ABT263-treated group. CTL, control; PARP, poly(ADP-ribose) polymerase.

Survivin, sense, 5'-GGACCACCG CATCTCTAC-3' and anti-sense, 5'-CAGCCTTCCAGCTCCTTG-3', with a 156-bp product (15); and $\beta$-actin, sense, 5'-GGATTCCTA TGTGGGCGACAG-3' and anti-sense, 5'-CGCTCGGTGAGGATCTTCATG-3', with a 438-bp product (15).

Isobologram analysis. The effects of two-drug combinations were assessed by isobologram analysis. The cells were treated with different concentrations of ABT263 $(0-20 \mu \mathrm{M})$ and PF4708671 (0-40 $\mu \mathrm{M})$. Combinations resulting in $25 \pm 1 \%$ cytotoxicity were expressed as a percentage of each single drug, alone, producing an equivalent level of cytotoxicity [fractional inhibitory concentrations $($ FIC) $=$ concentration of each drug in the combination/concentration of each drug alone]. When the sum of the FIC was 1, the combination was additive and the graph was expressed as a straight line; when the sum was $<1$, the combination was synergistic and the graph demonstrated a concave shape; and when the combination was $>1$, the combination was antagonistic and the graph demonstrated a convex shape.

Statistical analysis. Data are expressed as the mean \pm standard deviation of three independent experiments. A Student's t-test was performed to analyze differences between groups. All statistical analyses was performed using SigmaPlot software (version 11; Systat Software Inc., San Jose, CA, USA) and $\mathrm{P}<0.05$ was considered to indicate a statistically significant difference.

\section{Results}

Individual treatment with ABT263 or PF4708671 has no effect on cell death. Initially, the effects of ABT263 on BT474 on breast cancer cell apoptosis were examined. Apoptosis assays were performed using Annexin V/PI staining and western blot analysis of cleaved PARP expression. As indicated in Fig. 1A and B, treatment with $\leq 2 \mu \mathrm{M}$ ABT263 did not induce marked apoptotic cell death in the BT474 cells. Furthermore, protein expression levels of myeloid cell leukemia-1 [Mcl-1 (a Bcl-2-related protein)] were upregulated upon treatment with ABT-263, however, the expression levels of Bcl-2 and Bcl-xL did not change (Fig. 1B).

BT474 breast cancer cells are characterized by the overexpression of S6K1 (18). PF4708671 treatment suppressed S6K1 activity in the BT474 cells, as evidenced by the decreased phosphorylation of S6 at Ser240/244 (Fig. 1C). However, PF4708671 $(\leq 20 \mu \mathrm{M})$ resulted in no significant cell death (Fig. 1D). These data indicate that ABT263 or PF4708671 treatment alone is not sufficient to cause apoptosis.

Combined treatment with ABT263 and PF470867 synergistically induces cell death. The present study examined the effect of combined treatment with ABT263 and PF4708671 on the induction of cell death. Cell viability was reduced by $20 \%$ following treatment with $10 \mu \mathrm{M}$ PF4708671 and by $27 \%$ following treatment with $1 \mu \mathrm{M}$ ABT263. However, combination treatment with the two agents resulted in a 
A
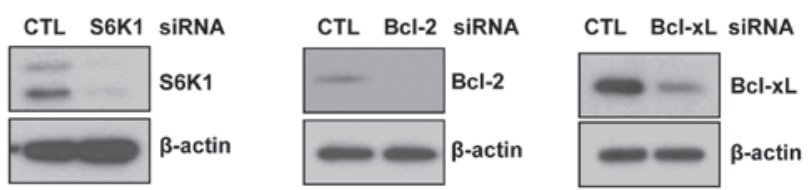

B

C
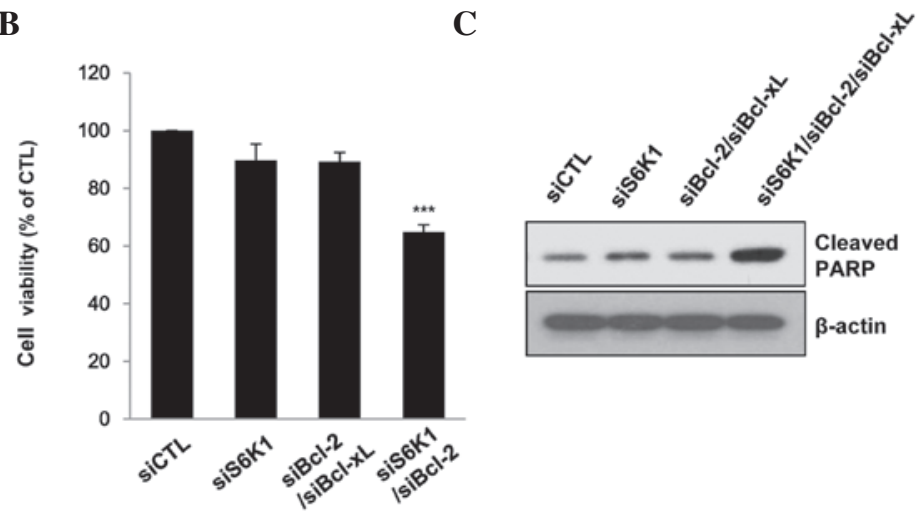

Figure 3. Silencing of S6K1 and Bcl-2/Bcl-xL induces cell death. (A-C) The BT474 cells were transiently transfected with the indicated target genes for $24 \mathrm{~h}$. (A and B) Expression levels of the indicated proteins were analyzed by western blotting and (C) cell viability was measured by performing a 3-(4,5-dimethylthiazolyl-2)-2,5-diphenyltetrazolium bromide assay. Data are presented as the means of triplicate samples, with error bars indicating the standard deviation from the mean.** $\mathrm{P}<0.001$ vs. siS6K1- or siBcl-2/Bcl-xL-treated group. CTL, control; S6K1, ribosomal S6 kinase 1; si, small interfering; Bcl-2, B-cell lymphoma-2; Bcl-xl, B-cell lymphoma-extra large; PARP, poly(ADP-ribose) polymerase.

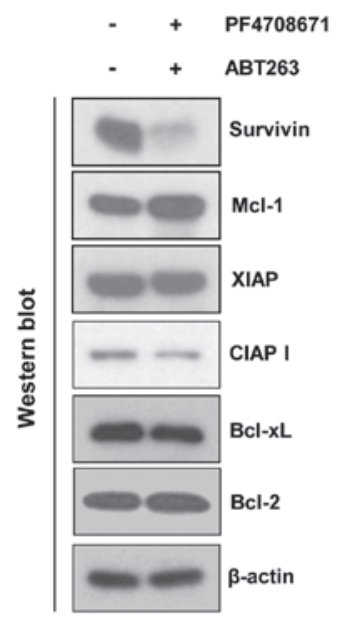

D

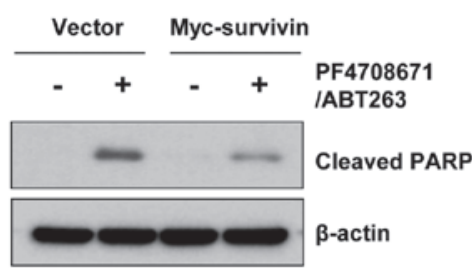

B

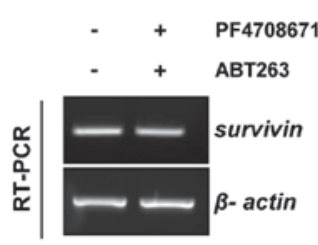

C

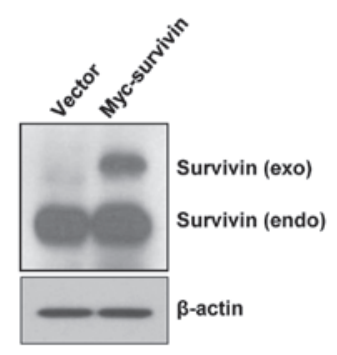

$\mathbf{E}$

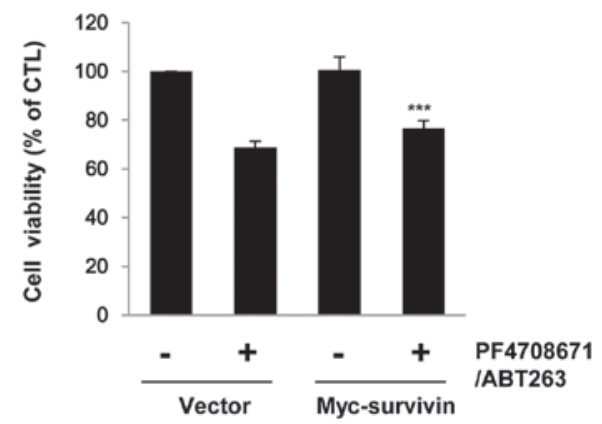

Figure 4. Downregulation of survivin expression by PF4708671 and ABT263 induces cell death. (A and B) BT474 cells were treated with $10 \mu \mathrm{M}$ PF4708671 and $1 \mu \mathrm{M} \mathrm{ABT} 263$ for $24 \mathrm{~h}$. (C-E) BT474 cells were transiently transfected with empty or myc-survivin vector for $24 \mathrm{~h}$, prior to treatment with $10 \mu \mathrm{M}$ PF4708671 and $1 \mu \mathrm{M} \mathrm{ABT} 263$ for $24 \mathrm{~h}$. (A, C and E) Protein expression levels were determined by western blotting and (B) mRNA expression levels were determined by RT-PCR. (D) Cell viability was analyzed by performing a 3-(4,5-dimethylthiazolyl-2)-2,5-diphenyltetrazolium bromide assay. The data are presented as the means of triplicate samples and the error bars indicate the standard deviation from the mean. ${ }^{* * *} \mathrm{P}<0.001$ vs. vector/PF4708671/ABT263-treated group. Mcl-1, myeloid cell leukemia-1; XIAP, X-linked inhibitor of apoptosis; cIAP1, cellular inhibitor of apoptosis 1; Bcl-2, B-cell lymphoma-2; Bcl-xl, B-cell lymphoma-extra large; RT-PCR, reverse transcription-polymerase chain reaction; CTL, control; PARP, poly(ADP-ribose) polymerase. 
significant reduction in cell viability $(\sim 60 \%$; $\mathrm{P}<0.01)$ (Fig. $2 \mathrm{~A})$ and the induction of apoptotic cell death, as examined by PARP cleavage and microscopy images of apoptosis (Fig. 2B and C). Furthermore, isobolographic analysis clarified the synergy between ABT263 and PF4708671 (Fig. 2D). These data indicate that combined treatment with ABT263 and PF4708671 synergistically induces cell death in BT474 breast cancer cells.

Silencing of S6K1 and Bcl-2/Bcl-xLinduces cell death. To further investigate the effect of the targeting of S6K1 and Bcl-2/Bcl-xL on BT474 cell death, the inhibitory effects of siRNAs targeting S6K1 and Bcl-2/Bcl-xL were investigated. The siRNAs caused marked knockdown of the target genes (Fig. 3A). The combined siRNA knockdown of S6K1 and Bcl-2/Bcl-xL significantly inhibited cell viability and induced PARP cleavage $(\mathrm{P}<0.001)$. By contrast, S6K1 or Bcl-2/Bcl-xL siRNA alone did not induce PARP cleavage (Fig. 3B) and exhibited marginal inhibitory effects on cell viability (Fig. 3C). These data indicate that the synergistic targeting of $\mathrm{S} 6 \mathrm{~K} 1$ and $\mathrm{Bcl}-2 / \mathrm{Bcl}-\mathrm{xL}$ may induce BT474 cell death.

Downregulation of survivin expression by PF4708671 and ABT263 induces cell death. The present study invesitgated the mechanisms involved in ABT263- and PF4708671-induced cell death. Combined treatment with ABT263 and PF4708671 appeared to have no effect on the expression of Bcl-2-related proteins, such as Bcl-2, Bcl-xL and Mcl-1. Notably, survivin protein, unlike inhibitor of apoptosis (IAP) family members, such as X-linked IAP (XIAP) and cellular IAP 1 (cIAP1), was significantly decreased in the presence of ABT263 and PF4708671 (Fig. 4A). However, combined treatment with ABT263 and PF4708671 did not modulate survivin mRNA expression levels (Fig. 4B), indicating that the observed changes in survivin expression following ABT263 and PF4708671 exposure did not involve transcriptional regulation.

To further clarify the role of survivin in ABT263 and PF4708671-induced cell death, empty or myc-survivin vectors were transiently transfected into treated BT474 cells prior to receiving combined ABT263 and PF4708671 treatment. The expression of myc-tagged survivin in the BT474 cells significantly suppressed the cell cytotoxicity induced by PF4708671 and ABT263 ( $<<0.001$; Fig. 4C and D), and attenuated the PARP cleavage induced by these inhibitors (Fig. 4E). These data indicate that the cell death induced by PF4708671 and ABT263 is at least in part mediated by the downregulation of survivin protein.

\section{Discussion}

The impairment of apoptosis is a hallmark of cancer and is key in the resistance to anticancer therapy. Tumors deploy diverse strategies to limit or evade apoptosis, frequently involving perturbation of the intrinsic Bcl-2 apoptotic pathway (19). As the overexpression of the pro-survival protein Bcl-2 is common in breast cancer (6), Bcl-2 may serve as a potential target for the treatment of this disease. ABT263 is an orally bioavailable small molecule inhibitor of Bcl-2 family proteins that binds with high affinity to Bcl-2 and Bcl-xL, and antagonizes their anti-apoptotic function (20). In the present study, ABT263 $(\leq 2 \mu \mathrm{M})$ treatment resulted in upregulated Mcl-1 expression, in agreement with a previous report (21). However, ABT263 $(\leq 2 \mu \mathrm{M})$ did not induce apoptotic cell death in BT474 breast cancer cells (Fig. 1). Preclinically, ABT263 has demonstrated limited activity in the majority of solid tumors containing breast cancer (22-24). However, when used in combination with other agents, such as rapamycin, rituximab, rituximab-cyclophosphamide, doxorubicin, vincristine and prednisolone, and bortezomib, ABT263 appeared to enhance their efficacy in leukemia and lymphoma models (23).

Our previous study indicated that phosphorylated S6K1, a downstream effector of mTORC1, may be effectively applied as a predictive marker for breast cancer cells (25). Indeed, S6K1 is frequently overexpressed in breast cancer cells (19). In the present study, although PF4708671 (an S6K1 inhibitor) suppressed S6K1 activity in the BT474 cells, PF4708671 $(\leq 20 \mu \mathrm{M})$ did not result in cell death (Fig. 1), indicating that PF4708671 alone is not sufficient to cause apoptosis. However, it was identified that combination treatment with ABT263 and PF4708671 synergistically induced BT474 cell death (Fig. 2). Furthermore, the combined inhibition of Bcl-2, Bcl-xL and S6K1 using siRNAs induced marked apoptosis (Fig. 3). These data indicate that ABT263 and PF4708671 in combination may be particularly effective treatment strategy for breast tumors with high levels of Bcl-2 and S6K1 expression.

ABT263 and PF4708671 combination treatment had no effect on the expression on anti-apoptotic Bcl-2 family proteins, such as Bcl-xL and Bcl-2, or IAP family proteins, such as XIAP and cIAP. However, ABT263 and PF4708671 notably induced decreases in survivin protein expression without affecting its mRNA expression. Furthermore, the overexpression of survivin attenuated the cell death induced by ABT263 and PF4708671. These results indicate that survivin may be involved in regulating the apoptosis induced by ABT263 and PF4708671.

In conclusion, data obtained in the present study indicates that ABT263 or PF4708671 treatment alone cannot induce apoptosis in breast cancer BT474 cells. However, combined treatment with ABT263 and PF4708671 appears to extensively induce cell death. Furthermore, the downregulation of survivin by ABT263 and PF4708671 treatment may induce cell death. Therefore, Bcl-2 and S6K1, which are frequently activated in breast cancer, may serve as potential targets in the treatment of patients with breast cancer.

\section{Acknowledgements}

The present study was supported by a grant from the Radiation Bio-Resource Research Program of the Korea Institute of Radiological and Medical Sciences (grant no. 740802).

\section{References}

1. Strasser A, Harris AW, Bath ML and Cory S: Novel primitive lymphoid tumours induced in transgenic mice by cooperation between myc and bcl-2. Nature 348: 331-333, 1990.

2. Green DR and Reed JC: Mitochondria and apoptosis. Science 281: 1309-1312, 1998.

3. Deng J, Carlson N, Takeyama K, Dal Cin P, Shipp M and Letai A: BH3 profiling identifies three distinct classes of apoptotic blocks to predict response to ABT-737 and conventional chemotherapeutic agents. Cancer Cell 12: 171-185, 2007.

4. Gross A, McDonnell JM and Korsmeyer SJ: BCL-2 family members and the mitochondria in apoptosis. Genes Dev 13: 1899-1911, 1999.

5. Callagy GM, Pharoah PD, Pinder SE, et al: Bcl-2 is a prognostic marker in breast cancer independently of the Nottingham Prognostic Index. Clin Cancer Res 12: 2468-2475, 2006. 
6. Dawson SJ, Makretsov N, Blows FM, et al: BCL2 in breast cancer: A favourable prognostic marker across molecular subtypes and independent of adjuvant therapy received. Br J Cancer 103: 668-675, 2010

7. Tse C, Shoemaker AR, Adickes J, et al: ABT-263: A potent and orally bioavailable Bcl-2 family inhibitor. Cancer Res 68: 3421-3428, 2008.

8. Rudin CM,Hann CL, Garon EB, et al: Phase II study of single-agent navitoclax (ABT-263) and biomarker correlates in patients with relapsed small cell lung cancer. Clin Cancer Res 18: 3163-3169, 2012.

9. Kamal A, Faazil S and Malik MS: Apoptosis-inducing agents: A patent review (2010-2013). Expert Opin Ther Pat 24: 339-354, 2014

10. Hong SE, Kim EK, Jin HO, et al: S6K1 inhibition enhances tamoxifen-induced cell death in MCF-7 cells through translational inhibition of Mcl-1 and survivin. Cell Biol Toxicology 29: 273-282, 2013.

11. Bärlund M, Forozan F, Kononen J, et al: Detecting activation of ribosomal protein S6 kinase by complementary DNA and tissue microarray analysis. J Natl Cancer Inst 92: 1252-1259, 2000.

12. Brugge J, Hung MC and Mills GB: A new mutational AKTivation in the PI3K pathway. Cancer Cell 12: 104-107, 2007.

13. Sinclair CS, Rowley M,Naderi A and Couch FJ: The 17q23 amplicon and breast cancer. Cancer Res Treat 78: 313-322, 2003.

14. Kim EK, Kim JH, Kim HA, et al: Phosphorylated S6 kinase-1: A breast cancer marker predicting resistance to neoadjuvant chemotherapy. Anticancer Res 33: 4073-4079, 2013.

15. Jin HO, Yoon SI, Seo SK, et al: Synergistic induction of apoptosis by sulindac and arsenic trioxide in human lung cancer A549 cells via reactive oxygen species-dependent down-regulation of survivin. Biochem Pharmacol 72: 1228-1236, 2006.

16. Lu TS, Yiao SY, Lim K, et al: Interpretation of biological and mechanical variations between the Lowry versus Bradford method for protein quantification. N Am J Med Sci 2: 325-328, 2010.
17. Jin HO, Lee YH, Kim HA, et al: Inhibition of vacuolar H+ ATPase enhances sensitivity to tamoxifen via up-regulation of CHOP in breast cancer cells. Biochem Biophys Res Commun 437: 463-468, 2013.

18. Noh WC, Mondesire WH, Peng J, et al: Determinants of rapamycin sensitivity in breast cancer cells. Clin Cancer Res 10: 1013-1023, 2004.

19. Strasser A, Cory S and Adams JM: Deciphering the rules of programmed cell death to improve therapy of cancer and other diseases. EMBO J 30: 3667-3683, 2011.

20. Vaillant F, Merino D, Lee L, et al: Targeting BCL-2 with the BH3 mimetic ABT-199 in estrogen receptor-positive breast cancer. Cancer Cell 24: 120-129, 2013.

21. Wang B, Ni Z, Dai X, et al: The Bcl-2/xL inhibitor ABT-263 increases the stability of Mcl-1 mRNA and protein in hepatocellular carcinoma cells. Mol Cancer 13: 98, 2014.

22. Shoemaker AR, Mitten MJ, Adickes J, et al: Activity of the Bcl-2 family inhibitor ABT-263 in a panel of small cell lung cancer xenograft models. Clin Cancer Res 14: 3268-3277, 2008.

23. Ackler S, Xiao Y, Mitten MJ, et al: ABT-263 and rapamycin act cooperatively to kill lymphoma cells in vitro and in vivo. Mol Cancer Ther 7: 3265-3274, 2008.

24. Lock R, Carol H, Houghton PJ, et al: Initial testing (stage 1) of the BH3 mimetic ABT-263 by the pediatric preclinical testing program. Pediatr Blood Cancer 50: 1181-1189, 2008.

25. Kim EK, Kim HA, Koh JS, et al: Phosphorylated S6K1 is a possible marker for endocrine therapy resistance in hormone receptor-positive breast cancer. Breast Cancer Res Treat 126: 93-99, 2011. 